\title{
UNDERSTANDING THE P CYGNI PROFILE
}

\author{
N. RONS \\ I.W.O.N.L. bursary holder \\ Astrofysisch Instituut, Vrije Universiteit Brussel, Pleinlaan 2, B-1050 Brussel, Belgium
}

\section{Introduction}

Narrow Absorption Components (NACs) have been detected in essentially all unsaturated P Cygni profiles of O-type stars (Howarth \& Prinja, 1989). Time-series of observations have revealed their migration through the profile's absorption trough (Prinja et al., 1987, Henrichs et al., 1988). The interpretation of the NACs is essential for the understanding of the dynamic structure of the winds of $\mathrm{O}$ stars and of the driving mechanisms behind it. The P Cygni profile substructures caused by isolated reverse and forward shocks were calculated in a 'ine only' approximation for a spherically symmetric, isothermal stellar wind. The Comoving Frame method was used for an accurate treatment of the complex radiation transfer in the shock environment. The underlying velocity structure follows a $\beta$-law (except in the innermost layers where a polynomial law was used) while the ionization was taken to be constant throughout the entire wind.

\section{Calculations and Interpretation}

Migration behaviour. Both reverse and forward shock calculations result in NACs which 'appear' at roughly half of the edge velocity, gain in strength as they migrate to gradually faint away as they approach the edge velocity (Fig. 1.). Shocks moving through the lower velocity regions of the wind cause some variation of the $P$ Cygni profile, but do not produce features recognizable as NACs in individual observations. Almost no influence of shocks is seen in the emission peak of the P Cygni profile. The main difference between NACs caused by reverse and forward shocks is in the order in which the increase and decrease in absorption occur in the profile with respect to the 'unshocked' model. It is not easy to distinguish between these two cases in the observed individual profiles and even in time-series this may not be straightforward as the NACs often are organized in a closely spaced recurrent scheme. The high velocity stage was found to be the best phase in which to identify the 'reverse' or 'forward' nature of the shocks in observed P Cygni profiles. After their migration the observed NACs remain at their highest velocity during a considerable lapse of time (Prinja, 1992). This behaviour is reproduced by the reverse shock models (Fig. 2), while the 


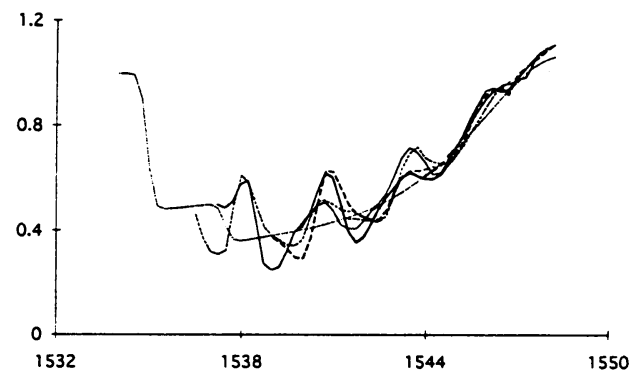

Fig. 1. Migration of theoretical NACs in the C IV $\lambda \lambda 1548.191550 .76$ doublet, caused by reverse (full line) and forward (dashed line) shocks. The dotted line is the 'unshocked' profile.

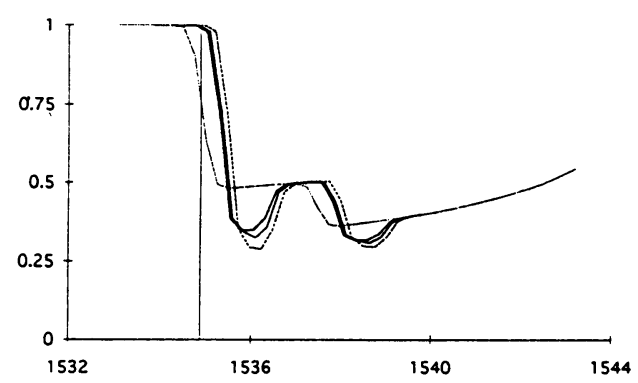

Fig. 2. Theoretical high velocity NACs in the C IV $\lambda \lambda 1548.191550 .76$ doublet, caused by reverse shocks. The dotted line is the 'unshocked' profile.

forward shock models result in features above the edge velocity disappearing more rapidly as the shocks move through the outer wind layers.

The NACs as a Source of Information. The NACs contain a wealth of information about the wind structure. In the case of reverse shocks, an NAC with a faint decreased absorption component points towards a shock with a sharp low velocity edge. A 'sharp edged' NAC points in the direction of relatively low thermal (and microturbulent) velocities. The width of the NAC is linked to the depth of the shock in the wind.

\section{References}

Henrichs, H.F., Kaper, L., Zwarthoed, G.A.A.: 1988, Ap. J. 317, 389

Henrichs, H.F., Kaper, L., Zwarthoed, G.A.A.: 1988, 'Rapid Variability in O Star Winds' in E.J. Rolfe, ed(s)., A Decade of UV Astronomy with IUE, Proc. Celebratory Symposium, GSFC, Greenbelt, USA, ESA SP-281, Vol. 2, 145

Howarth, I.D., Prinja, R.K.: 1989, Ap. J. Supp. Ser. 69, 527

Prinja, R.K., Howarth, I.D., Henrichs, H.F.: 1987, Ap. J. 317, 389

Prinja, R.K.: 1992, 'UV P Cygni Profile variability in O Stars' in L. Drissen, C. Leitherer, A. Nota, ed(s)., Nonisotropic and Variable Outflows from Stars, ASP Conference Series, Vol. 22, 167 